\title{
Prognostic and Predictive Value of P53, Bcl2, Rb and Egfr for Bladder Preservation in Invasive Bladder Carcinoma Treated by Trimodality Approach
}

\author{
Samy M. Al Gizawy ${ }^{1}$, Hoda H. Essa ${ }^{1}$, Abeer M. Refaiy ${ }^{2}$ and Gehan M. Elosaily ${ }^{2}$ \\ ${ }^{1}$ Departements of oncology and Pathology, ${ }^{2}$ Faculty of Medicine, Assuit university
}

Purpose: To update long-term results with selective organ preservation in invasive bladder cancer using aggressive transurethral resection of bladder tumor (TURBT) and radiochemotherapy (RCT) and to identify prognostic and predictive value of the biomarkers ;p53, pRB, BCL2 and EGFR.

Patients and Methods: Between 2000 and 2006, a total of 55 patients with T2-T3 bladder cancer were enrolled in 2 sequential bladder-sparing protocols including aggressive TURB and RCT. From September 2000 to May 2003, 25 patients (in protocol no. 1) were treated by TURBT followed by radiotherapy $46 \mathrm{~Gy}$ with concurrent cisplatin $20 \mathrm{mg} / \mathrm{m}^{2}$ day $1-5$, followed for complete and partial responders by radiotherapy 20 Gy with concurrent cisplatin (same dose) on the last five days. From December 2004 to April 2006, thirty patients were entered in protocol no. 2 that consisted of radiotherapy 60 Gy with concurrent Cisplatin $75 \mathrm{mg} /$ $\mathrm{m} 2$ q. 3 ws and Gemcitabine $300 \mathrm{mg} / \mathrm{m} 2$ D 1, 8 and 15 q. 3 ws for 2 cycles. In case of invasive residual tumor or recurrence, salvage cystectomy was recommended. All specimens were examined for expression of the biomarkers ( $\mathrm{p} 53, \mathrm{bcl} 2, \mathrm{Rb}$ and EGFR) using immunohistochemical staining.

Results: The median follow-up for all patients is 30 months (range 4-84), 38 months (range 9- 84) for patients in P1 and 22 months (4-54) for patients in P2. The actuarial 5-year OS were $58 \%$ (SE 5), 52\% (SE 7) and $61 \%$ (SE 6),for the whole series, $\mathrm{P} 1$ and $\mathrm{P} 2$ protocols respectively, $(\mathrm{P}=0.270)$. The corresponding figures for cancer specific survival (CSS) were $60 \%, 55 \%$ (SE 7) and 63\% (SE 4), (P=0.452). The 5-year actuarial OSB for all series, $\mathrm{P} 1$ and $\mathrm{P} 2$ protocols were $51 \%$ (SE 6), 46\% (SE 7) and 55\% (SE 9), respectively, ( $\mathrm{P}=0.323$ ). For all patients, altered expression of p53, bcl2, pRb and EGFR were detected in $(47.3 \%, 56.4 \%, 52.7 \%$ and $40 \%$ respectively The results of UVA showed that tumor stage and altered expression of pRB, BCL2 and EGFR were significantly associated with CSS and OS $(\mathrm{P}<0.05)$. There were no grade 4 toxicity and no treatmentrelated deaths.

Conclusion: Trimodality therapy to preserve the bladder is a therapeutic option that results in a high rate of long-term survivors retaining functional bladders in carefully selected patients. Patients with higher tumour stage and altered biomarkers; pRB, BCL2 and EGFR might not be candidate for bladder preserving approach.

Key words: Prognostic factor, P53, bladder presevation, bladder carcinoma

Corresponding Author: Hoda H. Essa

E-mail: hodahassanessa@yahoo.com

\section{INTRODUCTION}

The standard of care for localized muscle-invasive bladder cancer is radical cystectomy ${ }^{1}$. However, although this procedure is associated with excellent local control, it is not without risk of complications and, sometimes, poor quality of life results. Although significant improvements in continent urinary diversions have been made recently, there is still no substitute for the patient's own fully functional bladder.

In view of these problems, several clinical studies have been conducted using a bladder-sparing approach to the treatment of the disease. For example, radical transurethral resection (TUR) may be sufficient as monotherapy in some selected patients, as reported by Herr' ${ }^{2}$. However, in patients with deep muscle penetration who are treated exclusively with TUR, poor results have been observed. Similarly, neither chemotherapy (CT) nor radiation therapy (RT) alone results in significant local control ${ }^{3}$.

Therefore, recent strategies have combined the aforementioned interventions in an attempt to improve long-term survival and bladder preservation rates $^{4}$. Recently, clinical trials have been focused on the exploration of concurrent radiotherapy and chemotherapy as radiosensitizers (cisplatin-based chemotherapy and new drugs particularly gemcitabine) in order to further improve organ preservation in bladder cancer. Both agents (cisplatin and gemcitabine) have shown significant single-agent activity against urothelial tumors and are potent radiation sensitizers 5 . 
As demonstrated in several studies, the adequate selection of patients is a cornerstone for the success of a bladder sparing protocol ${ }^{3,5,6}$. Other than clinical stage, tumor-specific factors that have the ability to prognostically categorize patients with muscle-invasive bladder cancer into low- and high-risk groups for recurrence and survival and ultimately, delineate specific therapies, are needed ${ }^{7}$.

Over the past 2 decades, the molecular dissection of cancer has increased our understanding of the pathways that are altered in neoplastic cells. Protein expression profiling of bladder cancer offers an alternative means to distinguish aggressive tumor biology and may improve the accuracy of outcome prediction ${ }^{8}$. The majority of aggressive and invasive bladder carcinomas have alterations in the tumor suppressor gene products such as p53 and retinoblastoma $(\mathrm{Rb})^{9,10}$. An oncogene implicated in bladder cancer is epidermal growth factor receptor (EGFR) which was reported to be expressed in $70 \%$ of muscle-invasive bladder cancers and associated with poor prognosis ${ }^{11}$. Another oncogene that has been reported to be overexpressed in bladder is bcl- $2,{ }^{12}$.

In the present study we hypothesized that the expression of a panel of molecular markers (i.e. p53, pRB, BCL2 and EGFR) could help identify patients with muscle-invasive bladder cancer who are at increased risk for disease progression and therefore would not be candidates for a bladder-sparing approach.

The aim of the present study is to update long-term results with selective organ preservation in invasive bladder cancer using aggressive transurethral resection of bladder tumour (TURBT) and radiochemotherapy ( $\mathrm{RCT}$ ) and to identify prognostic and predictive value of the biomarkers ;p53, pRB, BCL2 and EGFR.

\section{PATIENTS AND METHODS}

Between 2000 and 2006, a total of 55 patients with T2-T3 bladder cancer were enrolled in 2 sequential bladder-sparing protocols including aggressive TURB and RCT. These two protocols were tested in two separate pilot studies. From September 2000 to May 2003, 25 patients, presented to radiotherapy department, South Egypt Cancer Institute (SECI), Assiut University, were included in protocol No. 1 (P1). From December 2004, to April 2006, thirty patients with invasive bladder cancer presented to urology and oncology outpatient clinics Assiut Univ. Hospital were enrolled in protocol No. 2 (P2).
The initial results of these two studies were reported in the years $2003^{13}$ and $2006^{14}$, respectively.

\section{Eligibility Criteria:}

In group 1, these criteria included patients with histopathologically proved invasive T.C.C. T2- T3b, N0, M0 and Karnofsky scale $>60 \%$, while in P2 they included patients with histopathologically proved invasive bladder cancer,(T2 - T3b, No - Mo) and Karnofsky scale $\geq$ $70 \%$. In both groups, the patients should have adequate hematological (platelets $>150.000 / \mathrm{mm} 2$, hemoglobin $>12 \mathrm{gm} / \mathrm{dl}$, leucocytes $>4.000 / \mathrm{mm} 2$ ), hepatic and renal functions (Creatinine clearance $>50 \mathrm{ml} / \mathrm{min}$. in P1) and adequate bladder capacity $\geq 350$ c.c. which was evaluated under anesthesia.

Exclusion criteria: in both groups, were patients with T4, N1-3 \&/or M1 and those who received BCG or intravesical chemotherapy.

Pretreatment Assessment: patients were assessed by history, clinical examination, complete blood count, blood chemistry, chest radiography and CT of the abdomen and pelvis.

\section{Treatment plan:}

Protocol no. 1 (P1): all patients were subjected to maximum transurethral resection of tumor (all visible tumors). Patients were then subjected to combined radio-chemotherapy, which were executed in two treatment phases. Phase I: external radiotherapy in the form of $46 \mathrm{~Gy} / 23$ fractions $/ 4 \frac{1}{2}$ weeks to whole pelvis with concurrent cisplatin $20 \mathrm{mg} / \mathrm{m}^{2} \mathrm{I}$.V infusion over two hours on the first five days. Patients with more than $50 \%$ response were shifted to phase II treatment. Patients with less than $50 \%$ response were considered for cystectomy. Phase II: 20 Gy / 10 fractions / 2 weeks to the bladder with concurrent cisplatin (same dose) on the last five days. After the end of treatment, patients with residual tumor underwent cystectomy and patients who had complete response were considered for follow up.

Protocol no. 2 (P2): Maximum resection of bladder tumour by transurethral resection followed by second look cystoscopy for resection of any residual tumor bed after two weeks or by partial cystectomy. After complete resection of bladder tumor, patients received combined chemo-radiotherapy 60 Gy of fractionated radiotherapy (200 cGy per setting) over 6 weeks with Cisplatin 75 $\mathrm{mg} / \mathrm{m}^{2} \mathrm{q} .3 \mathrm{ws}$ and Gemcitabine $300 \mathrm{mg} / \mathrm{m} 2 \mathrm{D} 1,8$ and 15 q. 3 ws for 2 cycles. Radiotherapy consisted also of two phases, phase I: a fractionated dose of $3600 \mathrm{cGy} / 18$ fractions/ 3.5 weeks was administered to the whole pelvis 
then phase II: in which $2400 \mathrm{cGy} / 12$ fractions/ 2.5 weeks was given to the bladder.

\section{Radiotherapy Techniques:}

The target volume in Phase I of both protocols included the bladder, the proximal urethra and the pelvic lymph nodes. The planning target volume (PTV) extends in the cranial-caudal dimension from L5-S1 interspace to the lower pole of the obturator foramen and $0.5-1 \mathrm{~cm}$ beyond the pelvic bones laterally. The anterior border lies 1-2 cm in front of the anterior bladder wall and the posterior border at the mid-rectum. Booster treatment was then used in phase II. In both protocols, it included the whole bladder and a margin of $2 \mathrm{~cm}$.

The patients were treated in supine position checked by laser lights. Fixation with thermoplastic shells was used in obese patients and in patients having redundant abdomen. IV contrast was used to localize the bladder.

Field arrangement for the proposed target volume was done by computerized planning system (P1) or manual method (P2) taking in consideration homogeneous distribution shape to the target volume and the tolerance dose to the critical organs. For whole pelvic irradiation (phase I), treatment was given with full bladder to displace the small bowel out of the pelvis. For phase II treatment, the patient was instructed to empty the bladder immediately before the treatment session to ensure that the bladder is inside the target volume. All patients were treated by photon linear accelerator 6 and/or $15 \mathrm{Mev}$. The total dose did not exceed $45 \mathrm{~Gy}$ and 55Gy to the femoral heads and the posterior rectal wall, respectively.

\section{Chemotherapy:}

All patients received concurrent chemotherapy immediately before radiotherapy fraction. Proper hydration and antiemetic (topisetron or ondansetron) were used before chemotherapy administration. Doses and schedules of both protocols are as labeled before.

\section{Histopathology and Immunohistochemistry:}

Tumor tissue samples were obtained by TUR preformed before treatment. Histology, tumor grade and stage were evaluated by reviewing of original pathology slides. Histologic grading was performed according to WHO 1973 criteria. Staging were done according to the TNM pathological staging system.

Immunohistochemistry for $\mathrm{p} 53, \mathrm{bcl} 2, \mathrm{Rb}$ and EGFR were performed according to manufacturing protocol. Tissue sections (4- $\mu \mathrm{m}$ thick) of formalin-fixed, paraffinembedded specimens were deparaffinized, rehydrated in graded alcohol and transferred to PBS (phosphate buffered saline, PH 7.6). The slides were rinsed twice with PBS, then endogenous peroxidase was blocked by the use of $3 \%$ hydrogen peroxide in methanol for $5 \mathrm{~min}$. antigen retrieval was done using microwave at $700 \mathrm{~W}$ for $20 \mathrm{~min}$ in citrate buffer for $\mathrm{p} 53$ and bcl2. for RB boiling in EDTA, ph 8 was done for 20 min. antigen retrival for EGFR was predigestion in protease enzyme for $5 \mathrm{~min}$. After cooling the slides were washed three times with PBS. The slides were incubated for $18 \mathrm{~h}$ (overnight) at $4 \mathrm{C}$ with primary antibody for $\mathrm{p} 53, \mathrm{bcl} 2, \mathrm{Rb}$ and EGFR (Thermo scientific CA, USA) at dilution of 1:50. The slides were then rinsed three times with PBS and incubated for $10 \mathrm{~min}$. at room temperature with the biotinylated goat antipolyvalent. The slides were rinsed with PBS for three times and incubated for $5 \mathrm{~min}$. with steptavidin peroxidase at room temperature. The sections were then washed three times with PBS and diaminibenzidine was applied for $5 \mathrm{~min}$ at room temperature. The slides then rinsed in D.W., counterstained with Mayer's hematoxylin, dehydrated, and then mounted. Negative control were obtained by ommiting the primary antibody.

A distinct brown nuclear staining was scored positive for p53 and Rb, membranocytoplasmic for EGFR and cytoplasmic for $\mathrm{bcl} 2$. Altered expression of $\mathrm{Rb}$ was defined as absence of nuclear staining, while p53, bcl2 and EGFR were positive if present in more than $10 \%$ of tumor cells.

\section{Follow-up:}

Evaluations consisted of a physical examination, blood counts and chemistry panels, urine analysis and cytology, cystoscopy and randomized biopsies from site of tumor 4-6 weeks after the end of treatment, every 3 months for 18 months and every 6 months thereafter.

Chest X-ray and abdominal and pelvis CT scans were performed

3 months after the completion of therapy and every 6 months for 18 months. then every year thereafter. Bone scans and other instrumental examinations were performed if indicated.

In patients who had persistent or recurrent tumor, additional treatment was recommended, such as TUR followed by intravesical therapy for superficial tumors or salvage cystectomy for muscle-invasive tumors if fit for surgery. All patients were followed up until Nov. 2009.

Primary endpoints were overall survival (OS), overall survival with bladder preservation (OSB), cancer specific survival(CSS) and late toxicity. Evaluation of late 
treatment-related toxicity was performed according to RTOG/EORTC criteria ${ }^{15}$.

\section{Statistical Methods of Analysis:}

Kaplan-Meier product-limit estimates were used to estimate the

probabilities of overall survival (OS), OS with bladder preservation

(OSB) was defined as the probability of remaining alive and with a preserved bladder. Disease free survival (DFS) was defined as the probability of remaining free of invasive bladder relapse, distant relapse, or death. For estimation of cancer specific survival (CSS), patients who died of unrelated causes were censored at death. Patients whose cause of death was unknown were assumed to have died of bladder cancer. Actuarial survival rates were calculated from the time of diagnosis to the time of the last follow-up visit or death. Univariate analysis (UVA) was performed using a Cox regression analysis to determine potential factors for risk of OS and $\mathrm{CSS}^{16}$.

\section{Tests of significance:}

Fisher exact test was used to study significance differences between variables.

$*$ P. value $\leq 0.05$ significant.

Statistical analysis was performed using SPSS for Windows version 16.0 (SPSS, Inc., Chicago, IL).

\section{RESULTS}

Patients and disease characteristics are summarized in Table (1).

Figure (1) showed immunohistochemical expression of four markers. For all patients, overexpression of p53, bcl 2 and EGFR were detected in $47.3 \%, 56.4 \%$ and $40 \%$, respectively. Loss of expression of $\mathrm{pRb}$ (altered) was detected in $52.7 \%$.

\section{Response:}

Patients who completed radiochemotherapy with bladder preservation were $45(81.8 \%), 18(72 \%)$ and $27(90 \%)$ for the whole series, P1 and P2 protocols, respectively. The remaining $10(18.2 \%)$ patients who had persistent disease after induction therapy included seven patients $(28 \%)$ subjected to salvage cystectomy in protocol No. 1 and 3 patients $(10 \%)$ in protocol No. 2 ; ; one patient died after end of treatment due to other cause rather than disease progression and 2 patients were having progressive disease, they were inoperable and they were treated with palliative chemotherapy.

\section{Outcome and Pattern of failure:}

The median follow-up for all patients is 30 months (range 4-84), 38 months (range 9- 84) for patients in $\mathrm{P} 1$ and 22 months (4-54) for patients in P2. Twenty nine patients $(52.7 \%)$ remain alive, 20 patients $(36.4 \%)$ died due to bladder tumor and 6 (11\%) from other nonrelated causes. Of the 45 patients who had CR, $5(9 \%)$ patients experienced superficial bladder relapse, $14(25.5 \%)$ patients developed muscle-invasive relapse and $9(16.4 \%)$ patients developed distant metastases. There were no significant differences regarding the incidence of superficial recurrences, muscle-invasive relapses, distant metastasis and deaths among the protocols.

Patients with superficial bladder relapse were treated conservatively with TUR and intravesical therapy. During follow up, three of them developed invasive recurrence, 2 of them underwent salvage cystectomy while the third patient refused. Patients who developed muscle-invasive relapse, $9(16.4 \%)$ of them were treated with salvage cystectomy while radical surgery could not be performed in the other 5 patients, because of poor performance status or patient refusal with median survival of 7 months. There was no pelvic recurrence for the patients who were subjected to salvage cystectomy.

\section{Survival and Bladder Preservation:}

The actuarial 5-year OS were $58 \%$ (SE 5), 52\% (SE 7) and 61\% (SE 6), for the whole series Figure (2), P1 and P2 protocols, respectively, Figure (3), $(\mathrm{P}=0.270)$, The corresponding figures for cancer specific survival (CSS) were 60\%, 55\% (SE 7) and 63\% (SE 4), $(\mathrm{P}=0.452)$. The 5-year actuarial OSB for all series, $\mathrm{P} 1$ and $\mathrm{P} 2$ protocols were $51 \%$ (SE 6), 46\% (SE 7) and 55\% (SE 9), respectively, $(\mathrm{P}=0.323)$. Thus, there was no significant difference in OS between both protocols.

\section{Prognostic factors:}

Univariate analysis was performed for the whole series to assess potential prognostic factors for OS and DFS, (Table 2). The results showed that PS, tumor stage and altered biomarkers: pRB, BCL2 and EGFR were significantly associated with DFS and OS.

\section{Toxicity: (Table 3)}

The main late toxicities are listed in Table. Seven patients $(13 \%)$ experienced late gastrointestinal toxicity (grade $\geq 2$ ) in the form of chronic diarrhea and tenesmus while $12(22 \%)$ patients developed late urinary toxicity (grade $\geq 2$ ) in the form of reduced bladder capacity, with less-than-voiding intervals $<2$ hours. There were no grade 4 toxicity and no treatment-related deaths. 
Table 1: Patients and disease characteristics in all cases

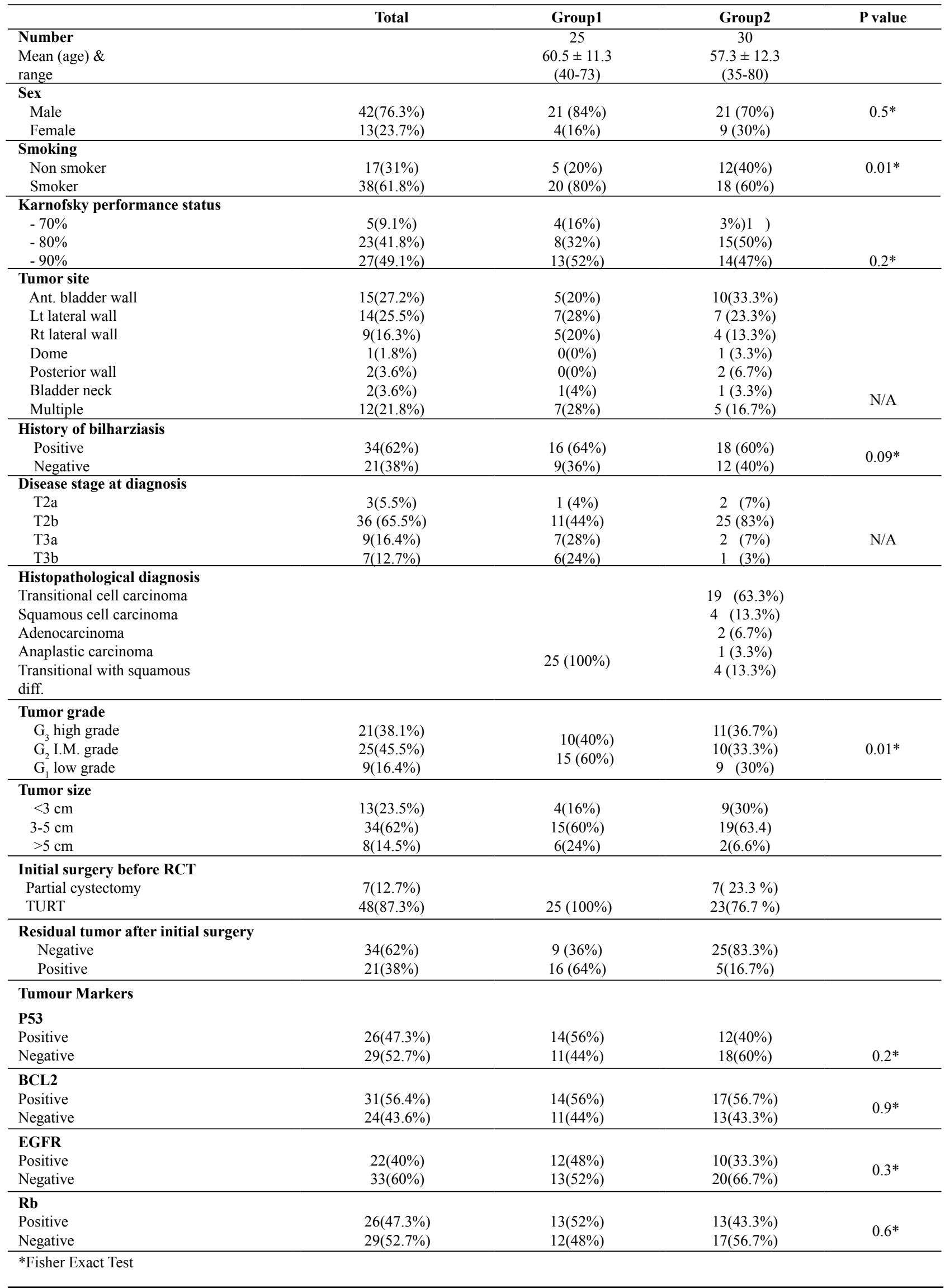


Table 2: Kaplan Meier's Univariate analysis of potential prognostic factors affecting disease free survival (A) and overall survival (B)

Table 2(A): Kaplan Meier's Univariate analysis of potential prognostic factors affecting disease free survival.

\begin{tabular}{|c|c|c|c|c|c|c|}
\hline & & \multirow{2}{*}{$\begin{array}{c}\text { Disease free } \\
\text { survival }\end{array}$} & \multirow{2}{*}{ Std. Error } & \multicolumn{2}{|c|}{ 95\% Confidence Interval } & \multirow{2}{*}{ P-value* } \\
\hline & & & & Lower Bound & Upper Bound & \\
\hline \multirow{2}{*}{ Sex } & female & 47.071 & 9.156 & 29.126 & 65.017 & \multirow{2}{*}{0.7} \\
\hline & male & 50.639 & 4.978 & 40.883 & 60.395 & \\
\hline \multirow{3}{*}{ P.S } & 70 & 25.200 & 6.089 & 13.266 & 37.134 & \multirow{3}{*}{0.02} \\
\hline & 80 & 40.435 & 7.047 & 26.623 & 54.246 & \\
\hline & 90 & 61.037 & 5.438 & 50.378 & 71.696 & \\
\hline \multirow{3}{*}{ Grade } & 1 & 48.667 & 4.704 & 39.448 & 57.886 & \multirow{3}{*}{0.15} \\
\hline & 2 & 48.846 & 6.403 & 36.297 & 61.395 & \\
\hline & 3 & 42.150 & 7.042 & 28.349 & 55.951 & \\
\hline \multirow{2}{*}{$\mathrm{R}$} & R0 & 51.035 & 5.007 & 41.221 & 60.849 & \multirow{2}{*}{0.6} \\
\hline & $\mathrm{R} 1$ & 45.833 & 9.474 & 27.265 & 64.401 & \\
\hline \multirow{2}{*}{ Hydronehrosis } & Yes & 54.400 & 14.506 & 25.968 & 82.832 & \multirow{2}{*}{0.8} \\
\hline & No & 49.831 & 4.779 & 40.465 & 59.198 & \\
\hline \multirow{2}{*}{ P53 } & negative & 58.621 & 6.110 & 46.644 & 70.597 & \multirow{2}{*}{0.06} \\
\hline & Positive & 49.192 & 5.546 & 28.323 & 50.062 & \\
\hline \multirow{2}{*}{$\mathrm{Bcl} 2$} & negative & 70.595 & 5.535 & 59.746 & 81.444 & \multirow{2}{*}{0.02} \\
\hline & Positive & 43.226 & 4.770 & 23.877 & 42.574 & \\
\hline \multirow{2}{*}{ EGFR } & negative & 55.313 & 5.907 & 43.735 & 66.892 & \multirow{2}{*}{0.03} \\
\hline & Positive & 31.545 & 6.260 & 29.276 & 53.815 & \\
\hline \multirow{2}{*}{$\mathrm{Rb}$} & negative & 51.138 & 5.962 & 39.452 & 62.824 & \multirow{2}{*}{0.04} \\
\hline & Positive & 38.474 & 6.502 & 35.730 & 61.219 & \\
\hline \multirow{2}{*}{ Tumor Stage } & $\mathrm{t} 2$ & 58.083 & 9.967 & 37.548 & 76.618 & \multirow{2}{*}{0.02} \\
\hline & t3 & 31.625 & 9.909 & 13.204 & 52.046 & \\
\hline
\end{tabular}

*Log Rank (Mantel-Cox)

Table2(B): Kaplan Meier's Univariate analysis of potential prognostic factors affecting overall survival.

\begin{tabular}{|c|c|c|c|c|c|c|}
\hline & & \multirow{2}{*}{ Overall survival } & \multirow{2}{*}{ Std. Error } & \multicolumn{2}{|c|}{ 95\% Confidence Interval } & \multirow{2}{*}{ P-value* } \\
\hline & & & & Lower Bound & Upper Bound & \\
\hline \multirow{2}{*}{ Sex } & female & 30.357 & 5.328 & 19.915 & 40.799 & \multirow{2}{*}{0.7} \\
\hline & male & 28.326 & 2.838 & 22.763 & 33.888 & \\
\hline \multirow{3}{*}{ P.S } & 70 & 16.600 & 4.248 & 8.273 & 24.927 & \multirow{3}{*}{0.03} \\
\hline & 80 & 19.174 & 2.453 & 14.366 & 23.982 & \\
\hline & 90 & 36.817 & 3.706 & 29.554 & 44.081 & \\
\hline \multirow{3}{*}{ Grade } & 1 & 27.111 & 3.852 & 19.561 & 34.661 & \multirow{3}{*}{0.17} \\
\hline & 2 & 30.516 & 3.861 & 22.947 & 38.084 & \\
\hline & 3 & 20.117 & 2.519 & 15.179 & 25.054 & \\
\hline \multirow{2}{*}{$\mathrm{R}$} & R0 & 30.733 & 3.127 & 24.603 & 36.862 & \multirow{2}{*}{0.5} \\
\hline & $\mathrm{R} 1$ & 21.117 & 3.577 & 14.106 & 28.127 & \\
\hline \multirow{2}{*}{ Hydronehrosis } & Yes & 22.000 & 2.926 & 16.266 & 27.734 & \multirow{2}{*}{0.8} \\
\hline & No & 30.171 & 2.973 & 24.343 & 35.999 & \\
\hline \multirow{2}{*}{ P53 } & Positive & 25.172 & 2.277 & 20.709 & 29.636 & \multirow{2}{*}{0.06} \\
\hline & negative & 18.048 & 3.567 & 18.057 & 32.038 & \\
\hline \multirow{2}{*}{$\mathrm{Bcl} 2$} & Positive & 43.245 & 3.500 & 36.386 & 50.105 & \multirow{2}{*}{0.02} \\
\hline & negative & 27.262 & 1.715 & 13.900 & 20.624 & \\
\hline \multirow{2}{*}{ EGFR } & Positive & 33.994 & 3.681 & 26.779 & 41.209 & \multirow{2}{*}{0.04} \\
\hline & negative & 20.727 & 2.188 & 16.439 & 25.016 & \\
\hline \multirow{2}{*}{$\mathrm{Rb}$} & Positive & 33.221 & 2.069 & 19.166 & 27.275 & \multirow{4}{*}{0.04} \\
\hline & negative & 20.847 & 3.952 & 22.100 & 37.594 & \\
\hline \multirow{2}{*}{ Tumor stage } & t2 & 34.036 & 3.891 & 26.411 & 41.662 & \\
\hline & $\mathrm{t} 3$ & 16.875 & 2.730 & 11.524 & 22.226 & \\
\hline
\end{tabular}

*Log Rank (Mantel-Cox) 
Kasr-El-Aini Journal Of Clinical Oncology And Nuclear Medicine

Vol. 7 | No. 1-2 2011

Prognostic and Predictive Value of P53, Bcl2, Rb

Table 3: Summary of late complications.

\begin{tabular}{lcccc}
\hline & Total $(\mathbf{n}=\mathbf{5 5})$ & P 1 $(\mathbf{n}=\mathbf{2 5})$ & P 2 (n= 30) & P value \\
\hline Urinary grade $\geq 2$ & $12(22 \%)$ & $5(20 \%)$ & $7(23.3 \%)$ & 0.76 \\
\hline Grade 2 & $10(18 \%)$ & $4(16 \%)$ & $6(20 \%)$ & 0.74 \\
\hline Grade 3 & $2(3.6 \%)$ & $1(4 \%)$ & $1(3.3 \%)$ & 0.77 \\
\hline Grade 4 & 0 & 0 & 0 & 0.60 \\
\hline Intestinal grade $\geq 2$ & $7(13 \%)$ & $3(12 \%)$ & $4(13.3 \%)$ & 0.58 \\
\hline Grade 2 & $5(9 \%)$ & $2(8 \%)$ & $3(10 \%)$ & 0.77 \\
\hline Grade 3 & $2(3.6 \%)$ & $1(4 \%)$ & $1(3.3 \%)$ & 0 \\
\hline Grade 4 & 0 & 0 & &
\end{tabular}

Figure 1: Immunohistochemical expression of four markers (overexpression of p53, bcl2, EGFR and pRb-altered).

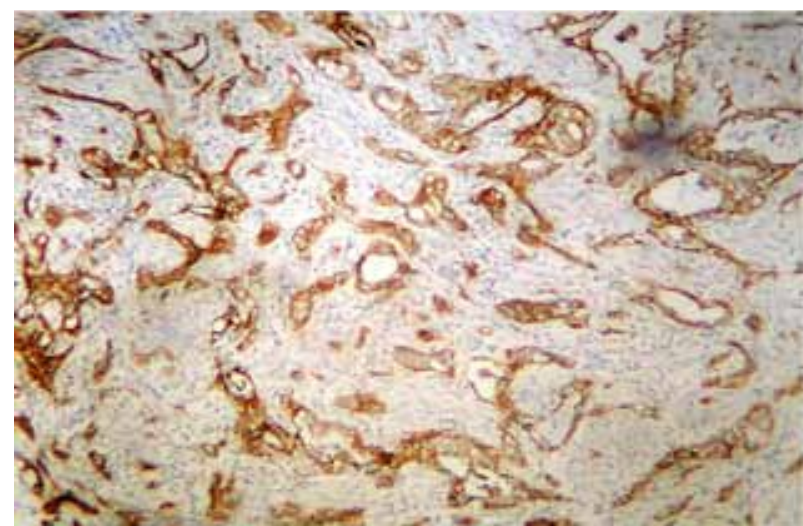

Figure 1 (A): overexpression of p53.

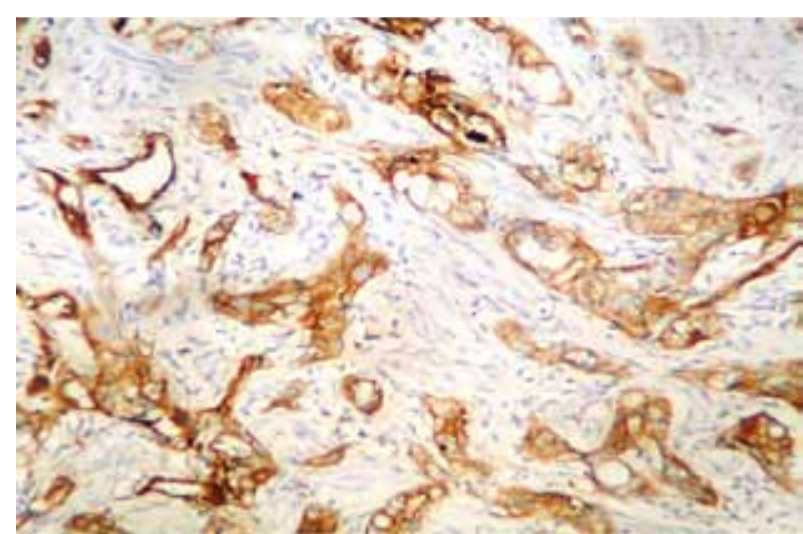

Figure 1 B: overexpression of bcl2.

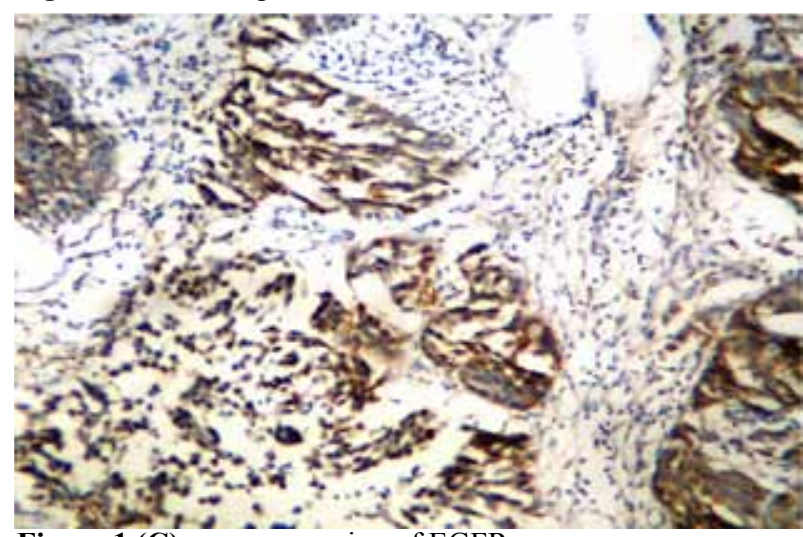

Figure 1 (C): overexpression of EGFR

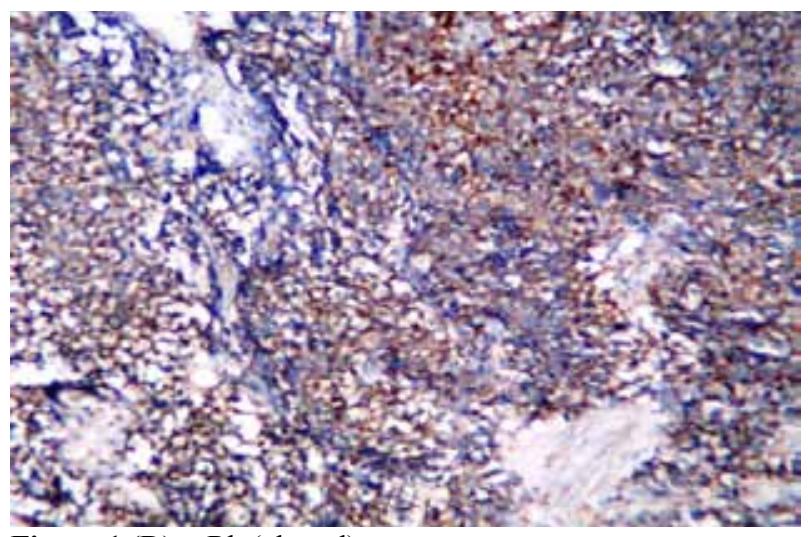

Figure 1 (D): pRb (altered)

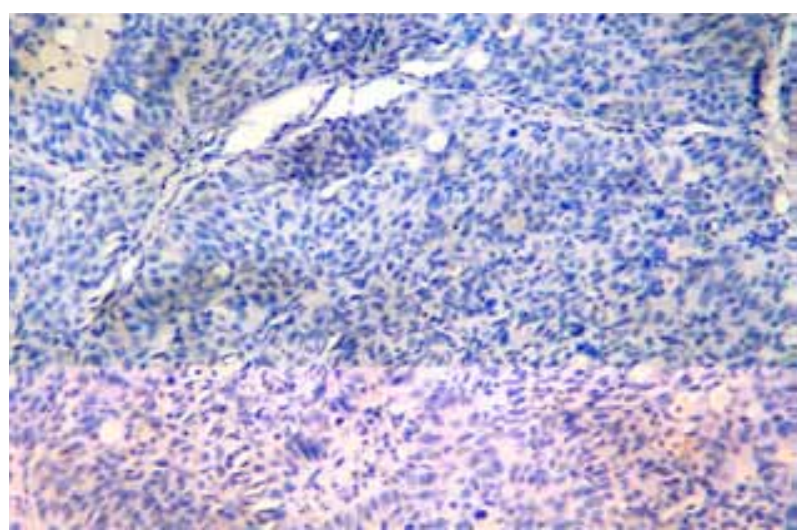

\section{Figure 1 E}

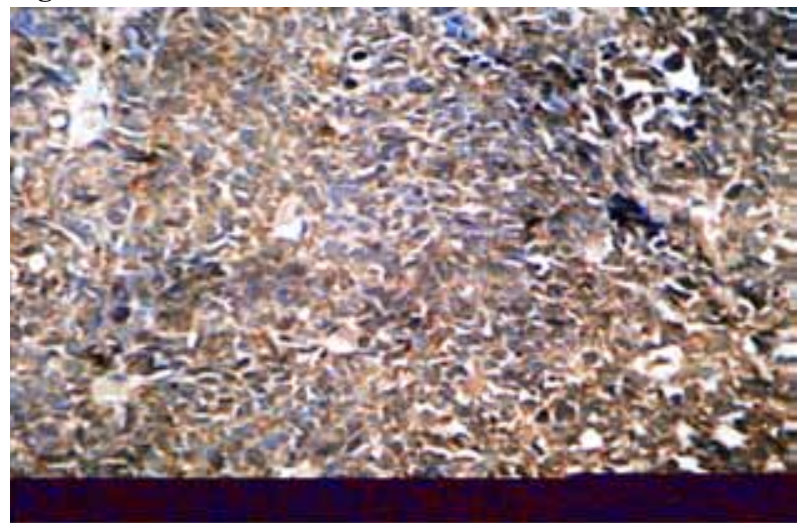

Figure 1 F 
Figure 2: Kaplan-Meier overall survival (OS) of all patients.

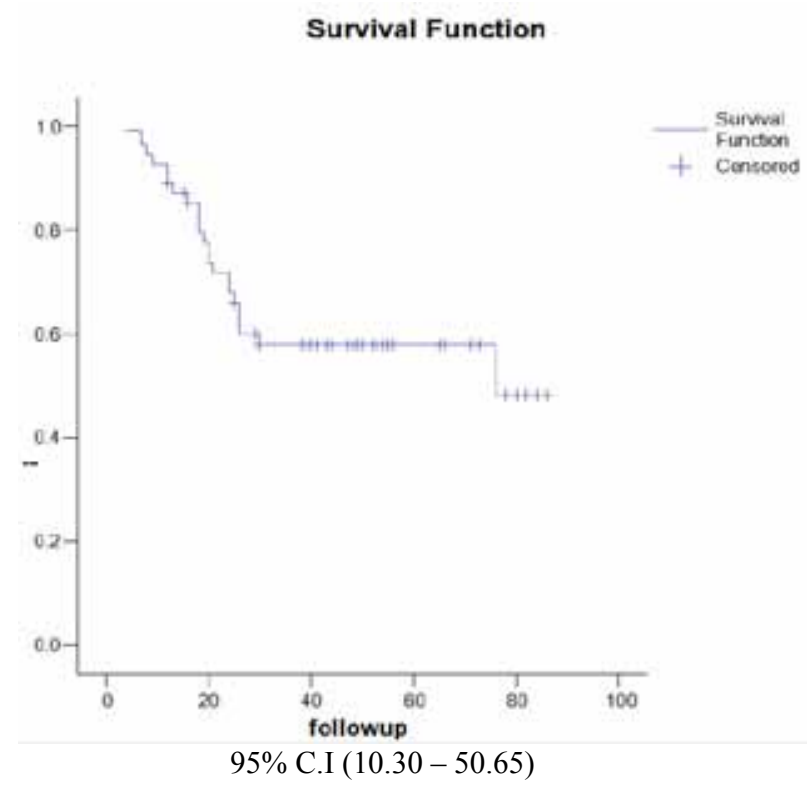

Figure 2: Kaplan-Meier overall survival (OS) of all patients.

\section{DISCUSSION}

The present study has updated our experience in our university hospitals with transurethral resection of the bladder and chemoradiotherapy approach to bladder preservation in invasive bladder cancer. More experience with organ-sparing treatment is very important for the optimization of the treatment modalities and the proper selection of patients who will most probably benefit from the respective treatment alternatives ${ }^{5}$.

For all patients, OS, CSS and OSB rates at 5 years reached $58 \%, 60 \%$ and $51 \%$, respectively with no significant difference between the two protocols. These results, similar to those reported by the best reported series, support the use of bladder-sparing treatment in selected patients as a safe and an effective alternative to radical cystectomy ${ }^{5,17-21}$.

Despite these encouraging results, the outcome of the organ-sparing approach needs to be compared with the surgical standard. Unfortunately, primary cystectomy has not been tested against combined-modality treatment in randomized trials ${ }^{4,22}$.

Our findings that $T$ classification after the initial bladder preserving surgery was an independent prognostic factor is in agreement with the extensive evidence from most of the studies ${ }^{3,5,6}$. More recently, Cobo et al. ${ }^{23}$ reported on 29 patients who underwent TUR and received neoadjuvant CT and RCT followed by consolidation RT when a CR was achieved. Again, those investigators reported that early-stage disease was predictive of the best outcome. However, our finding that the initial resection status
Probability

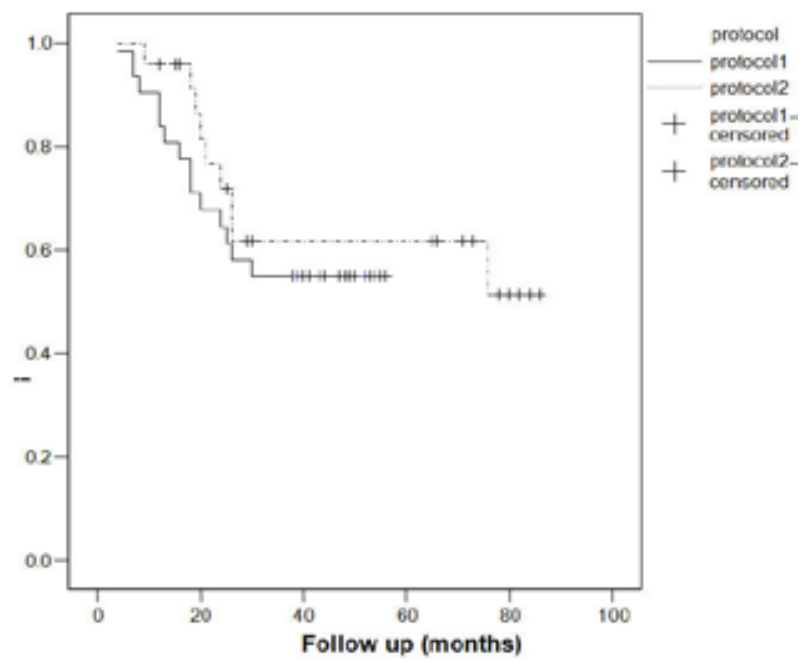

Figure 3: Kaplan-Meier overall survival (OS) by treatment protocol:

95\% CI for protocol 1: $(19.2-32.16)$

$95 \% \mathrm{CI}$ for protocol $2:(10.3-29.34)$

and hydronephrosis did not have prognostic impact in bladder preserving approach are not in agreement with that reported by similar studies ${ }^{4,5,23}$.

Regardless of pathologic stage, altered biomarkers; pRB, BCL2 and EGFR correlate significantly with poor prognosis and recurrence of the disease. In agreement with these findings, recent research has shown significantly different response rates of muscle-invasive bladder cancers treated with radiation and concurrent chemotherapy, based on the expression of $\mathrm{EGFR}^{24}$. Pollack et al. ${ }^{25}$ have also described a relation between altered expression of the pRB and bcl-2 genes and the local response of patients to preoperative radiotherapy. On the contrary to results of other studies ${ }^{8,26}, \mathrm{p} 53$ mutation did not correlate with clinical outcome of our patients. There are several explanations for these discrepancies including different patient population (heterogeneity, limited size), diverse tumor types, the types of drugs used and differences in methods of assessment of p53 alterations $s^{27,28}$. Large multiinstitutional prospective studies are needed to clarify the role of p53 and other biomarkers in muscle-invasive bladder cancer.

When irradiating bladder tumors, the organs at risk are normal bladder tissue, bowel and the rectum. The incidences of grade $3 \mathrm{GU}$ and GI late complications were acceptably low $3.6 \%$ for each, with no grade 4 toxicities. In reported series, cystectomy for bladder contracture has been rare: $2 \%$ of patients in the Erlangen series ${ }^{29}$ and none in the Massachusetts General Hospital series ${ }^{30}$. The majority of patients in our series have retained good bladder function and quality of life, as also reported by Zietman et al., ${ }^{21}$ who performed functional urodynamic 
studies in and administered a validated QoL questionnaire to 49 long-term survivors of their trimodality protocols.

\section{CONCLUSION}

Trimodality therapy to preserve the bladder is a therapeutic option that results in a high rate of long-term survivors retaining functional bladders. Even if radical cystectomy remains the standard of care for muscleinvasive bladder cancer, a combined-modality treatment can be offered as a reasonable option to carefully selected patients. Patients with higher tumour stage and altered biomarkers; pRB, BCL2 and EGFR might not be candidate for bladder preserving approach.

Further evaluation of molecular prognostic factors is essential to advancing the role of combined modality treatment in the management of this disease. Finally, only randomized trials comparing directly cystectomy with a multimodality bladder-preserving approach can determine whether they have equally effective results.

\section{REFERENCES}

1. Stein JP, Skinner DG. Radical cystectomy for invasive bladder cancer: Long-term results of a standard procedure. World J.Urol. 2006 Aug;24(3):296-304.

2. Herr HW. Transurethral resection of muscle-invasive bladder cancer: 10-year outcome. J.Clin.Oncol. 2001 Jan 1;19(1):89-93.

3. Kuczyk M, Turkeri L, Hammerer P, Ravery V. Is there a role for bladder preserving strategies in the treatment of muscle-invasive bladder cancer? Eur.Urol. 2003 Jul;44(1):57-64.

4. Perdonà S, Autorino R, Damiano R, De Sio M, Morrica B, Gallo L, et al. Bladder-sparing, combinedmodality approach for muscle-invasive bladder cancer: A multi-institutional, long-term experience. Cancer 2008;112(1):76-83.

5. Zapatero A, Martin de Vidales C, Arellano R, Bocardo G, Pérez M, Ríos P. Updated results of bladder-sparing trimodality approach for invasive bladder cancer. Urol. Oncol. 2010;28(4):368-74.

6. Milosevic M, Gospodarowicz M, Zietman A, Abbas F, Haustermans K, Moonen L, et al. Radiotherapy for bladder cancer. Urology 2007;69(1 Suppl.):80-92.

7. Maluf FC, Cordon Cardo C, Verbel DA, Satagopan JM, Boyle MG, Herr H, et al. Assessing interactions between $\mathrm{mdm}-2, \mathrm{p} 53$ and bcl-2 as prognostic variables in muscle-invasive bladder cancer treated with neoadjuvant chemotherapy followed by locoregional surgical treatment. Ann.Oncol. 2006 Nov;17(11):1677-86.

8. Shariat SF, Karakiewicz PI, Ashfaq R, Lerner SP, Palapattu GS, Cote RJ, et al. Multiple biomarkers improve prediction of bladder cancer recurrence and mortality in patients undergoing cystectomy. Cancer 2008;112(2):31525.

9. Mitra AP, Birkhahn M, Cote RJ. p53 and retinoblastoma pathways in bladder cancer. World J.Urol. 2007;25(6):56371.

10. Bellamy CO, Malcomson R, Wyllie A. The role of p53 in apoptosis and cancer. In: Martin SJ, editor. Apoptosis and cancer. Basel: Karger Landes systems; 1997. p. 67-71.

11. Colquhoun AJ, Mchugh LA, Tulchinsky E, Kriajevska M, Mellon JK. Combination treatment with ionising radiation and gefitinib ('Iressa', ZD1839), an Epidermal Growth Factor Receptor (EGFR) inhibitor, significantly inhibits bladder cancer cell growth in vitro and in vivo. J.Radiat. Res. 2007;48(5):351-60.

12. Cho HJ, Kim JK, Kim KD, Yoon HK, Cho MY, Park YP, et al. Upregulation of Bcl-2 is associated with cisplatinresistance via inhibition of Bax translocation in human bladder cancer cells. Cancer Lett. 2006 Jun 8;237(1):5666.

13. Ahmed MA. Bladder preserving approach by chemoradiotherapy in patients with muscle invading transitional cell carcinoma. Assiut University: South Egypt Cancer Institute (SECI); 2003.

14. Mekkawy MA, Eid SS, El Taher AM, Mostafa HG, Abdul Aziz AM. Phase II study of concurrent chemoradiotherapy for bladder preservation in treatment of invasive bladder cancer. Kasr El Aini J.Oncol.Nucl.Med. 2006 Nov;2(39):373.

15. Cox JD, Stetz J, Pajak TF. Toxicity criteria of the Radiation Therapy Oncology Group (RTOG) and the European Organization for Research and Treatment of Cancer (EORTC). Int.J.Radiat.Oncol.Biol.Phys. 1995 Mar 30;31(5):1341-6.

16. Cox DR. Regression models and life-tables. J.R.Stat. Soc.B 1972;34(2):187-220.

17. Rodel C, Weiss C, Sauer R. Organ preservation by combined modality treatment in bladder cancer: The European perspective. Semin.Radiat.Oncol. 2005 Jan;15(1):28-35.

18. Fernando SA, Sandler HM. Multimodality bladder preservation therapy for muscle-invasive bladder tumors. Semin.Oncol. 2007;34(2):129-34.

19. Chung PW, Bristow RG, Milosevic MF, Yi QL, Jewett MA, Warde PR, et al. Long-term outcome of radiationbased conservation therapy for invasive bladder cancer. Urol.Oncol. 2007 Jul-Aug;25(4):303-9.

20. Weiss C, Engehausen DG, Krause FS, Papadopoulos T, Dunst J, Sauer R, et al. Radiochemotherapy with cisplatin and 5-fluorouracil after transurethral surgery in patients with bladder cancer. Int.J.Radiat.Oncol.Biol.Phys. 2007 Jul 15;68(4):1072-80.

21. Zietman AL, Sacco D, Skowronski U, Gomery P, Kaufman DS, Clark JA, et al. Organ conservation in invasive bladder cancer by transurethral resection, chemotherapy and radiation: Results of a urodynamic and quality of life study 
on long-term survivors. J.Urol. 2003 Nov;170(5):1772-6.

22. Given RW, Parsons JT, McCarley D, Wajsman Z. Bladdersparing multimodality treatment of muscle-invasive bladder cancer: A five-year follow-up. Urology 1995 Oct:46(4):499-504; discussion 504-5.

23. Cobo M, Delgado R, Gil S, Herruzo I, Víctor B, Carabante $\mathrm{F}$, et al. Conservative treatment with transurethral resection, neoadjuvant chemotherapy followed by radiochemotherapy in stage T2-3 transitional bladder cancer. Clin.Transl.Oncol. 2006;8(12):903-11.

24. Chakravarti A, Winter K, Wu CL, Kaufman D, Hammond E, Parliament $\mathrm{M}$, et al. Expression of the epidermal growth factor receptor and Her-2 are predictors of favorable outcome and reduced complete response rates, respectively, in patients with muscle-invading bladder cancers treated by concurrent radiation and cisplatinbased chemotherapy: A report from the Radiation Therapy Oncology Group. Int.J.Radiat.Oncol.Biol.Phys. 2005 Jun 1;62(2):309-17.

25. Pollack A, Wu CS, Czerniak B, Zagars GK, Benedict WF, McDonnell TJ. Abnormal bcl-2 and pRb expression are independent correlates of radiation response in muscle-invasive bladder cancer. Clin.Cancer Res. 1997 Oct;3(10):1823-9.

26. Malats N, Bustos A, Nascimento CM, Fernandez F, Rivas $\mathrm{M}$, Puente D, et al. P53 as a prognostic marker for bladder cancer: A meta-analysis and review. Lancet Oncol. 2005 Sep;6(9):678-86.

27. Garcia del Muro X, Condom E, Vigues F, Castellsague X, Figueras A, Munoz J, et al. p53 and p21 Expression levels predict organ preservation and survival in invasive bladder carcinoma treated with a combined-modality approach. Cancer 2004 May 1;100(9):1859-67.

28. Schmitz Drager BJ, Goebell PJ, Ebert T, Fradet Y. p53 immunohistochemistry as a prognostic marker in bladder cancer. Playground for urology scientists? Eur.Urol. 2000 Dec;38(6):691-9; discussion 700.

29. Rodel C, Weiss C, Sauer R. Trimodality treatment and selective organ preservation for bladder cancer. J.Clin. Oncol. 2006 Dec 10;24(35):5536-44.

30. Mak RH, Zietman AL, Heney NM, Kaufman DS, Shipley WU. Bladder preservation: Optimizing radiotherapy and integrated treatment strategies. BJU Int. 2008;102(9B):1345-53. 